\title{
Cinacalcet versus standard treatment for chronic kidney disease: a protocol for a systematic review and meta-analysis
}

\author{
Nigar Sekercioglu*, Jason W. Busse ${ }^{1,3,4}$, Reem A. Mustafa ${ }^{1,5}$, Gordon H. Guyatt ${ }^{1,2}$ and Lehana Thabane $e^{1,6,7,8,9}$
}

\begin{abstract}
Background: Chronic kidney disease-mineral and bone disorders (CKD-MBD) have been associated with poor health outcomes, including diminished quality and length of life. Standard management for CKD-MBD includes phosphate-restricted diet, active vitamin D, vitamin D analogs, and phosphate binders. Persistently elevated parathyroid hormone (PTH) levels may require the addition of Cinacalcet hydrochloride (cinacalcet) which sensitizes calcium receptors on the parathyroid glands. The objective of this systematic review is to compare the effect of cinacalcet versus standard treatment in patients with CKD-MBD.

Methods/design: Data sources will include MEDLINE, EMBASE, the Cochrane Register of Controlled Trials, and Web of Science from 1996 to June 2015. Teams of two reviewers will, independently and in duplicate, screen titles and abstracts and potentially eligible full text reports to determine eligibility, and subsequently abstract data and assess risk of bias in eligible trials. We will calculate the effect estimates (risk ratios or mean differences) and $95 \%$ confidence intervals, as well as statistical measures of variability in results across studies using random effect models for patient-important and intermediate outcomes. We will use the GRADE (Grading of Recommendations, Assessment, Development and Evaluation) approach to rate the quality of evidence about estimates of effect on an outcome-by-outcome basis. We will present our results with a GRADE summary table.
\end{abstract}

Discussion: Our review will explore the effect of cinacalcet versus standard treatment in patients with CKD-MBD. The results of this systematic review will help guide management of this patient population, and identify targets for future research.

Systematic review registration: PROSPERO CRD42015020318http://www.crd.york.ac.uk/PROSPERO/ display_record.asp?ID=CRD42015020318.

Keywords: Chronic kidney disease, Mineral and bone disorders, Secondary hyperparathyroidism, Calcimimetic agents

\section{Background}

Chronic kidney disease-mineral and bone disorder (CKD-MBD) is a systematic condition defined by three components: (1) extra skeletal calcifications; (2) abnormal metabolism of serum calcium, phosphorus, parathyroid hormone (PTH), and vitamin D; and (3) abnormal bone metabolism, including bone turnover, mineralization, linear growth, and reduced strength [1]. CKD-MBD is also associated with extravascular calcifications, increased

\footnotetext{
*Correspondence: sekercn@mcmaster.ca

'Department of Clinical Epidemiology and Biostatistics, McMaster University,

1280 Main Street West, Hamilton, Ontario L8S 4K1, Canada

Full list of author information is available at the end of the article
}

cardiovascular events, and mortality [1]. The pathogenesis of CKD-MBD involves receptor level problems, including vitamin $\mathrm{D}$ receptors and calcium-sensing receptors $[2,3]$. Abnormal serum calcium, phosphorus, $\mathrm{PTH}$, and vitamin $\mathrm{D}$ levels also contribute to the development of the disease process [2-4].

Parathyroid gland hyperplasia is a common finding in patients with CKD-MBD, and proliferation in parathyroid cells leads to increase in parathyroid gland secretion. This leads to secondary hyperparathyroidism (SHPT) which is associated with elevated serum PTH levels [5]. A consensus exists regarding the need for CKD-MBD treatment to maintain guideline recommended targets for calcium, 
phosphorus, and $\mathrm{PTH}$ in the presumption that meeting these targets will improve quality and quantity of life $[3,6-8]$.

In the management of CKD-MBD, recommendations suggest that patients should, in addition to phosphaterestricted diet, receive active vitamin $\mathrm{D}$ and phosphate binders [3, 6, 9]. Medical management of persistent elevated PTH requires the use of calcimimetic agents. Cinacalcet hydrochloride (cinacalcet) is a second generation calcimimetic agent used to sensitize calcium receptors on the parathyroid glands $[10,11]$. This leads to decreased PTH synthesis and secretion [10, 11]. Cinacalcet is a positive allosteric modulator that interacts with non-calcium binding sites [2]. Although this therapy has a very wide applicability, including parathyroid cancers and primary and secondary hyperparathyroidism, cost is a major drawback that limits utilization [12].

In 2004, the US Food and Drug Administration approved cinacalcet for uncontrolled SHPT in CKD-MBD patients. The medication is not recommended for predialysis patients because of the potential risks for severe hypocalcemia and phosphate increase due to the low PTH levels $[6,13,14]$. The total cost of cinacalcet per patient with CKD-MBD ranges from $\$ 4000$ to $\$ 23,500$ per year in Canada.

CKD-MBD has been associated with increased mortality, and reduced bone and cardiovascular health [15]. Prior systematic reviews have found that cinacalcet treatment reduces the rate of parathyroidectomy, fracture, and hospitalization due to cardiovascular events $[16,17]$; the effect on mortality has not been established [18-20]. However, prior reviews have not included trials published after February 2013 and only included adult CKD patients.

We will update search covering more databases and the pediatric patient population with transparent quality assessment of findings. The consequences of CKD-MBD and pharmacokinetic and pharmacodynamic properties of cinacalcet may differ between the adult and pediatric patient populations. We will explore for a subgroup effect on this basis (adult versus children) and, if the test of interaction is significant, we will present results separately for these populations.

The objective of this systematic review is to evaluate the impact of cinacalcet treatment as compared to standard treatment alone or standard treatment plus placebo in patients with CKD-MBD and uncontrolled SHPT on patient-important outcomes, including cardiovascular events, fractures, parathyroidectomy, symptomatic hypotension, cardiovascular mortality, all-cause mortality, and intermediate outcomes-achieving Kidney Disease Outcome Quality Initiative targets (K/DOQI).

\section{Methods/design}

\section{Data sources and search strategy}

We will search the MEDLINE, EMBASE, and Cochrane Register of Controlled Trials and Web of Science databases from 1996 until June 2015 without any restrictions. We will use controlled vocabulary and text words to search all databases. We will scan the bibliographies of all prior systematic reviews and meta-analyses as well as all eligible primary studies for additional relevant articles. We will search for conference proceedings from 2010 to 2015 using the Web of Science and abstracts presented at recent annual meetings (the American Society of Nephrology, National Kidney Foundation, International Society of Nephrology and European Renal Association-European Dialysis and Transplant Association). Please refer to Table 1 for the full search strategy.

\section{Eligibility criteria}

We will limit the studies included in this review to RCTs evaluating the effectiveness and safety of cinacalcet for the treatment of secondary HPT. The comparator will be the standard therapy alone or standard therapy plus placebo. The standard therapy includes phosphate binders and/or active vitamin $\mathrm{D}$. This review will explore the effectiveness of cinacalcet treatment on the following outcomes: cardiovascular events, fractures, parathyroidectomy, symptomatic hypotension, cardiovascular mortality, all-cause mortality, end-of-treatment alkaline phosphatase, end-of-treatment PTH levels (any measure), end-oftreatment serum calcium concentrations $(\mathrm{mg} / \mathrm{dL}$ or $\mathrm{mmol} / \mathrm{L}$ ), end-of-treatment serum phosphorus concentrations $(\mathrm{mg} / \mathrm{dL}$ or $\mathrm{mmol} / \mathrm{L})$, end-of-treatment calcium $\mathrm{x}$ phosphorus product $\left(\mathrm{mg}^{2} / \mathrm{dL}^{2}\right)$. We will not employ any restrictions for age. We will not include studies that the primary objective of which is to determine optimal dosing of cinacalcet and economic evaluation of cinacalcet treatment.

\section{Study selection}

Teams of two investigators will independently screen each unique title and abstract that our literature search identifies. If either reviewer identifies a citation as potentially relevant, we will obtain the full text of the article. Two reviewers will independently determine the eligibility of all studies that undergo full text evaluation. We will measure the inter-rater agreement for full text eligibility and assessment of the risk of bias using the kappa statistic [21]. Values of kappa between 0.40 and 0.59 have been considered to reflect fair agreement, between 0.60 and 0.74 to reflect good agreement and $\geq 0.75$ to reflect excellent agreement [21]. Disagreements will be resolved through discussion between the reviewers or through adjudication with a third party if necessary. 
Table 1 Search strategy for the MEDLINE, EMBASE and Cochrane Central Register of Controlled Trials databases

MEDLINE

Set History

$1 \quad$ (((kidney* or nephro* or renal or home or peritoneal or intermittent or chronic or extracorporeal or ambulatory) adj2 (haemodialys* or hemodialys* or dialys*)) or hemorenodialysis or hemodialyse or (APD).ti,ab.

2 renal dialysis/ or hemodialysis, home/ or peritoneal dialysis/ or peritoneal dialysis, continuous ambulatory/

3 renal insufficiency, chronic/ or kidney failure, chronic/

4 (((chronic or "end-stage" or "end stage") adj3 (kidney* or renal or nephro*) adj3 (insufficien* or disease $\left.^{*}\right)$ ) or esrd).ti,ab.

5 frasier syndrome/ or ("frasier* syndrome*" or (frasier* adj2 syndrome*)).mp.

6 renalosteodystrophy/ or ((renal or kidney* or nephro*) adj2 (osteodystroph* or ricket*)).mp.

7 azotemia/ or azotemi*.mp.

8 uremia/ or uremi*.mp

9 or/1-1

10 Calcimimetic Agents/ or (Calcimimetic* or amg073 or cinacalcet or "krn 1493" or krn1493 or mimpara or parareg or regpara or sensipar).mp.

119 and 10

12 controlled clinical trial.pt. or controlled clinical trials as topic/ or meta analysis.pt. ormeta analysis as topic/ or multicentre study.pt. or multicenter studies as topic/ or randomized controlled trial.pt. or randomized controlled trials as topic/ or pragmatic clinical trial.pt. or Pragmatic Clinical Trials as Topic/ or ((preference or practical or pragmatic or "real world" or naturalistic) adj5 trial*).ti,ab. or Comparative Effectiveness Research/ or ((comparative adj2 effectiveness) or (CER adj5 (research* or

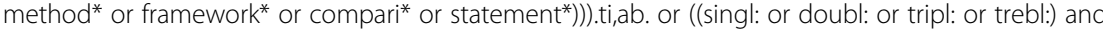
(mask: or blind:)).ti,ab. or ((random: adj5 trial:) or rct or rets).ti,ab.

1311 and 12

EMBASE

Set History

$1 \quad$ (((kidney* or nephro* or renal or home or peritoneal or intermittent or chronic or extracorporeal or ambulatory) adj2 (haemodialys* or hemodialys* or dialys*)) or hemorenodialysis or hemodialyse or (APD).ti,ab.

2 renal replacement therapy/ or continuous ambulatory peritoneal dialysis/ or continuous renal replacement therapy/ or extended daily dialysis/ or hemodialysis/ or home dialysis/ or peritoneal dialysis/

3 kidney failure/ or chronic kidney failure/

4 (((chronic or "end-stage" or "end stage") adj3 (kidney* or renal or nephro*) adj3 (insufficien* or disease*)) or esrd).ti,ab.

5 frasier syndrome/ or ("frasier* syndrome*" or (frasier* adj2 syndrome*)).mp.

6 renalosteodystrophy/ or ((renal or kidney* or nephro*) adj2 (osteodystroph* or ricket*)).mp. (7537)

7 azotemia/ or azotemi*.mp.

8 uremia/ or uremi*.mp.

9 Or/1-8

10 Calcimimetic Agent/ or cinacalcet/ or (Calcimimetic* or amg073 or cinacalcet or "krn 1493" or krn1493 or mimpara or parareg or regpara or sensipar).mp.

119 and 10

12 ct.fs. or controlled clinical trial.pt. or controlled clinical trial/ or meta analysis/ or multicenter study/ or randomized controlled trial.pt. or randomized controlled trial/ or double blind procedure/ or single-blind procedure/ or triple blind procedure/ or ((preference or practical or pragmatic or "real world" or naturalistic) adj5 trial*).ti,ab. or comparative effectiveness/ or ((comparative adj2 effectiveness) or (CER adj5 (research* or method* or framework* or compari* or statement*))).ti,ab. or ((random: adj5 trial:) or rct or rcts).ti,ab. or ((singl: or doubl: or tripl: or trebl:) and (mask: or blind:)).ti,ab.

$13 \quad 11$ and 12
Comments

Dialysis textword search Terms

Dialysis subject Terms

Chronic kidney disease subject terms

Chronic kidney disease textword terms

Syndrome subject and textword terms

Chronic kidney disease subject or textword Terms

Azotemia subject or textword Terms

Uremia disease subject or textword Terms

Kidney disease terms

Cinacalcet subject and textword search

Terms

Base clinical set

Therapy Study design methodologies

Comments

Dialysis textword search Terms

Dialysis subject Terms

Chronic kidney disease subject terms

Chronic kidney disease textword terms

Syndrome subject and textword terms

Chronic kidney disease subject or textword Terms

Azotemia subject or textword Terms

Uremia disease subject or textword Terms

Kidney disease terms

Cinacalcet subject and textword search Terms

Base clinical set

Therapy Study design methodologies 
Table 1 Search strategy for the MEDLINE, EMBASE and Cochrane Central Register of Controlled Trials databases (Continued)

EBM Reviews - Cochrane Central Register of Controlled Trials

Set History

1 ((kidney* or nephro* or renal or home or peritoneal) adj2 (haemodialys* or hemodialys* or dialys*)).ti,ab. or renal dialysis/ or hemodialysis, home/ or peritoneal dialysis/ or peritoneal dialysis, continuous ambulatory/ or renal insufficiency, chronic/ or kidney failure, chronic/ or (((chronic or "end-stage" or "end stage") adj3 (kidney* or renal or nephro*) adj3 (insufficien* or disease*)) or esrd).ti,ab. or frasier syndrome/ or ("frasier* syndrome*" or (frasier* adj2 syndrome*)).mp. or renal osteodystrophy/ or ((renal or kidney* or nephro*) adj2 (osteodystroph* or ricket*)).mp. or azotemia/ or azotemi*.mp. or uremia/ or uremi*.mp. or (((kidney* or nephro* or renal or home or peritoneal or intermittent or chronic or extracorporeal or ambulatory) adj2 (haemodialys* or hemodialys* or dialys*)) or hemorenodialysis or hemodialyse or (APD).ti,ab. or kidney failure/ or chronic kidney failure/ or frasier syndrome/ or renal osteodystrophy/ or uremia/ or (((chronic or "end-stage" or "end stage") adj3 (kidney* or renal or nephro*) adj3 (insufficien* or disease*)) or esrd).ti,ab. or kidney failure/ or chronic kidney failure/ or frasier syndrome/ or ("frasier* syndrome*" or (frasier* adj2 syndrome*)).mp. or renal osteodystrophy/ or ((renal or kidney* or nephro*) adj2 (osteodystroph* or ricket*)).mp. or azotemia/ or azotemi*.mp. or uremia/ or uremi*.mp.

2 Calcimimetic Agent/ or cinacalcet/ or Calcimimetic Agents/ or (Calcimimetic* or amg073 or cinacalcet or "krn 1493" or krn1493 or mimpara or parareg or regpara or sensipar).mp.

Comments

Dialysis, chronic kidney diseases Subject or textword search Terms

Cinacalcet subject and textword search Terms

Base clinical set

\section{Data abstraction}

Using a standardized data collection form, two reviewers will collect the following information from each study: the author, date of publication, eligibility criteria, summary of baseline characteristics of the participants, number of participants in each arm at study onset and completion, duration of the trial, and treatment effects, including effectiveness and safety. We will resolve disagreements by discussion.

\section{Quality assessment of individual studies}

A modified version of the Cochrane risk for bias tool (http://distillercer.com/resources/) will be employed by two independent reviewers in order to assess risk of bias on an outcome-by-outcome basis for all eligible trials. The assessment will include the following components: adequacy of sequence generation, allocation sequence concealment, level of blinding, incomplete outcome data, loss to follow-up, and stopping early for benefit or futility [22]. Reviewers will input response options of "definitely yes", "probably yes", "probably no", and "definitely no" for each of the domains, with definitely yes and probably yes ultimately assigned low risk of bias and definitely no and probably no assigned high risk of bias [23]. Reviewers will resolve disagreements by discussion, and one arbitrator will adjudicate unresolved disagreements.

We will use to assess the quality of evidence for each outcome using the GRADE (Grading of Recommendations, Assessment, Development and Evaluation) rating system [24]. In the GRADE system of rating quality of evidence for each outcome, randomized trials begin as high quality evidence, but may be rated down by one or more of five categories of limitations [25]. We will use the GRADE methodology to rate certainty in effect estimates and quality of evidence for each outcome as high, moderate, low, or very low [25]. We will use the detailed GRADE guidance to assess the overall risk of bias, imprecision, inconsistency, indirectness, and publication bias and summarize results with a GRADE summary table [26]. The quality of evidence will be rated down, if we determine risk of bias as well as large imprecision, inconsistency, indirectness, and publication bias.

With the respect to precision, we will assess the optimal information size (OIS; the number of patients generated by a conventional sample size calculation for a single trial) and the width of the $95 \%$ CIs. GRADE guidance notes that meta-analyses of small trials can provide evidence of benefit with $95 \%$ CIs that appear to convincingly exclude no effect; however, the results of reviews of such studies have often been subsequently refuted by larger trials [27, 28]. To address this potential concern in cases in which our meta-analysis suggests benefit but the sample size is less than the OIS, we will rate down imprecision. For the purposes of calculating the OIS, we will assume, for binary variables a relative risk reduction or increase (delta) of $25 \%$, an alpha of 0.05 , and a beta of 0.20 , and a median baseline risk from the available studies. In addition, we will examine the width of $95 \%$ CIs and verify if $95 \%$ CIs overlap clinical decision thresholds for each outcome.

In addition to assessment of statistical heterogeneity, the degree of proximity in point estimates and overlap in $95 \%$ confidence intervals (95\% CI) will be examined to assess consistency between studies. Differences in methodology, study populations, outcomes, and quality and quantity of interventions will be considered to evaluate directness.

For each outcome, we will assess publication bias by visually observing asymmetry of the funnel plot for each outcome [29, 30]. We will follow published guidance and construct funnel plots only for outcomes with $\geq 10$ 
trials. Publication bias will be considered as unlikely unless the effect measure is asymmetrically distributed around the pooled effect [29].

After considering these reasons for rating down, we will judge the quality of evidence for each outcome as follows: "high" quality of evidence (we are very confident that the true effect lies close to that of the estimate of the effect); "moderate" quality of evidence (we are moderately confident in the effect estimate and the true effect is likely to be close to the estimate of the effect, but there is a possibility that it is substantially different); "low" quality of evidence (our confidence in the effect estimate is limited and the true effect may be substantially different from the estimate of the effect); and "very low" quality of evidence (we have very little confidence in the effect estimate and the true effect is likely to be substantially different from the estimate of the effect) [25].

\section{Data synthesis and statistical analysis}

We will report descriptive statistics as proportions for categorical variables and mean or medians for the continuous variables. We will calculate pooled risk ratios (RRs) and the associated $95 \%$ CI for each outcome using random effects models by applying the MantelHaenszel method. Absolute effects and the associated $95 \%$ CI will be calculated by multiplying pooled RRs and $95 \% \mathrm{CI}$ by the pooled control rate of outcomes. Statistical heterogeneity will be assessed by the chi-square test and $I^{2}$ statistic. All data analyses will be performed using Stata (StataCorp. 2013. Stata Statistical Software: Release 13. College Station, TX: StataCorp LP).

\section{Dealing with missing participant data for dichotomous outcomes: sensitivity analyses}

We will conduct a complete-case analysis for our primary analysis and perform sensitivity analyses to address the robustness of our findings with respect to the missing data. We plan to use plausible worst-case scenario for the missing trial-level data. The extent to which point estimates and $95 \%$ CIs differ in these sensitivity analyses will determine whether we rate down for risk of bias [30, 31].

\section{Assessment of heterogeneity and subgroup analyses}

Our estimates of study heterogeneity will be informed using the $p$ value for the chi-square test for homogeneity and the $I^{2}$ statistic where 0-40\% may be unimportant heterogeneity, 30-60\% moderate, 50-90\% substantial, and $75-100 \%$ considerable heterogeneity [32]. If the $I^{2}$ statistic indicates a value $>50 \%$, we will explore heterogeneity. We will employ random effects meta-regression and include mean age, the mean baseline serum PTH concentration, trial duration, and stages of CKD (dialysis versus non-dialysis) in our univariate linear models.
We will employ subgroup analysis for the pediatric and adult patient populations. If the interaction test suggests a significant subgroup effect, we will report effect estimates for each subgroup separately.

\section{Presentation of results}

We will present the results of our meta-analyses in an evidence profile that will provide a succinct, easily digestible presentation of quality of evidence, and magnitude of effects [26]. Our evidence profile will be constructed to include the following elements: a measure of the typical burden of these outcomes (e.g., control group, estimated risk; if appropriate studies are available, we will use the baseline risk for population-based observational studies); a measure of the absolute risk difference between the risks with and without intervention; the relative magnitude of effect; the numbers of participants and studies addressing these outcomes; a GRADE rating of the overall confidence in estimate of effect for each outcome and any reasons for rating down the confidence [32].

\section{Discussion}

We expect to provide an objective assessment of effectiveness and safety of cinacalcet in patients with CKD-MBD. We will examine the impact of cinacalcet on patientimportant outcomes. In addition, we will explore the quality of the existing evidence in both adult and pediatric patient populations with CKD-MBD. This will be the first systematic review that includes both pediatric and adult CKD patient populations in the assessment of cinacalcet and patient important outcomes available in the literature. We will disseminate our results in local meetings and in a peer-reviewed publication.

\section{Strengths and limitations of this study}

The methods of our proposed review are state of the art, including explicit eligibility criteria, a comprehensive search, independent duplicate assessment of eligibility, and the use of the GRADE approach to assessing quality of evidence of effect including independent duplicate assessment of risk of bias, precision, consistency, directness, and publication bias. Our protocol represents a model for systematic review methods. Our results are likely to be limited by limitations in the primary studies.

\footnotetext{
Abbreviations

Ca: calcium; CKD-MBD: chronic kidney disease-mineral and bone disorders; GRADE: Grading of Recommendations, Assessment, Development and Evaluation; OIS: optimal information size; P: phosphate; PTH: parathyroid hormone; RCT: randomized controlled trail; RR: risk ratios; SHPT: secondary hyperparathyroidism.
}

Competing interests

The authors declare that they have no competing interests. 


\section{Authors' contributions}

All authors participated in the preparation of the manuscript and agreed to the submitted version of the paper. NS contributed to the conception and design of the protocol and drafted the manuscript. LT, GG, RAM, and JWB contributed to the conception, design, and revision of the manuscript. All authors read and approved the final manuscript.

\section{Acknowledgements}

We have not received any funding for this review.

\section{Author details}

'Department of Clinical Epidemiology and Biostatistics, McMaster University, 1280 Main Street West, Hamilton, Ontario L8S 4K1, Canada. ${ }^{2}$ Department of Medicine, McMaster University, 1280 Main Street West, Hamilton, Ontario L8S 4K1, Canada. ${ }^{3}$ The Michael G. DeGroote Institute for Pain Research and Care, McMaster University, Hamilton, Canada. ${ }^{4}$ Department of Anesthesia, McMaster University, Hamilton, Canada. ${ }^{5}$ Departments of Internal Medicine and Biomedical \& Health Informatics, University of Missouri-Kansas City, Kansas City, USA. ${ }^{6}$ Department of Pediatrics and Anesthesia, McMaster University, Hamilton, Canada. ${ }^{7}$ Centre for Evaluation of Medicine, St Joseph's Healthcare Hamilton, Hamilton, Canada. ${ }^{8}$ Biostatistics Unit, Father Sean O'Sullivan Research Centre, St Joseph's Healthcare, 3rd Floor, Martha Wing, 50 Charlton Avenue East, Hamilton, Ontario L8N 4A6, Canada. ${ }^{9}$ Population Health Research Institute, Hamilton Health Sciences, Hamilton, Canada.

Received: 31 July 2015 Accepted: 21 December 2015

Published online: 04 January 2016

\section{References}

1. Moe S, Drüeke T, Cunningham J, Goodman W, Martin K, Olgaard K, et al. Definition, evaluation, and classification of renal osteodystrophy: a position statement from Kidney Disease: Improving Global Outcomes (KDIGO). Kidney Int. 2006;69(11):1945-53

2. Omata M, Fukagawa M, Kakuta T. Vascular calcification-pathological mechanism and clinical application-. Vascular calcification in chronic kidney disease-mineral and bone disorder (CKD-MBD). Clin Calcium. 2015;25(5):645-53.

3. Wetmore JB, Quarles LD. Calcimimetics or vitamin D analogs for suppressing parathyroid hormone in end-stage renal disease: time for a paradigm shift? Nat Clin Pract Nephrol. 2009;5(1):24-33.

4. Cunningham J, Locatelli F, Rodriguez M. Secondary hyperparathyroidism: pathogenesis, disease progression, and therapeutic options. Clin J Am Soc Nephrol CJASN. 2011;6(4):913-21.

5. Block GA, Martin KJ, de Francisco AL, Turner SA, Avram MM, Suranyi MG, et al. Cinacalcet for secondary hyperparathyroidism in patients receiving hemodialysis. N Engl J Med. 2004;350(15):1516-25.

6. Drueke TB. Cell biology of parathyroid gland hyperplasia in chronic renal failure. J Am Soc Nephrol. 2000;11(6):1141-52.

7. KDIGO clinical practice guideline for the diagnosis, evaluation, prevention, and treatment of chronic kidney disease-mineral and bone disorder (CKD-MBD). Kidney Int Suppl, 2009(113): p. S1-130. doi: 10.1038/ki.2009.188.

8. Uhlig K, Berns JS, Kestenbaum B, Kumar R, Leonard MB, Martin KJ, et al. KDOQI US commentary on the $2009 \mathrm{KDIGO}$ clinical practice guideline for the diagnosis, evaluation, and treatment of CKD-mineral and bone disorder (CKD-MBD). Am J Kidney Dis. 2010;55(5):773-99.doi: 10.1053/j.ajkd.2010.02.340

9. Eknoyan G, Levin A, Levin NW. K/DOQI clinical practice guidelines for bone metabolism and disease in chronic kidney disease. Am J Kidney Dis, 2003. 42(4 Suppl 3): p. S1-201

10. Hammerland LG, Garrett JE, Hung BC, Levinthal C, Nemeth EF. Allosteric activation of the $\mathrm{Ca} 2+$ receptor expressed in Xenopus laevis oocytes by NPS 467 or NPS 568. Mol Pharmacol. 1998;53(6):1083-8.

11. Nemeth EF, Steffey ME, Hammerland LG, Hung BC, Van Wagenen BC, DelMar EG, et al. Calcimimetics with potent and selective activity on the parathyroid calcium receptor. Proc Natl Acad Sci U S A. 1998;95(7):4040-5.

12. Qunibi WY. Cardiovascular calcification in nondialyzed patients with chronic kidney disease. Semin Dial., 2007. 20(2): p. 134-8.

13. Charytan C, Coburn JW, Chonchol M, Herman J, Lien YH, Liu W, et al. Cinacalcet hydrochloride is an effective treatment for secondary hyperparathyroidism in patients with CKD not receiving dialysis. Am J Kidney Dis. 2005;46(1):58-67.
14. Chonchol M, Locatelli F, Abboud HE, Charytan C, de Francisco AL, Jolly S, et al. A randomized, double-blind, placebo-controlled study to assess the efficacy and safety of cinacalcet $\mathrm{HCl}$ in participants with CKD not receiving dialysis. Am J Kidney Dis. 2009;53(2):197-207.

15. Noordzij M, Korevaar JC, Boeschoten EW, Dekker FW, Bos WJ, Krediet RT, et al. The Kidney Disease Outcomes Quality Initiative (K/DOQI) guideline for bone metabolism and disease in CKD: association with mortality in dialysis patients. Am J Kidney Dis. 2005;46(5):925-32.

16. Palmer SC, Nistor I, Craig JC, Pellegrini F, Messa P, Tonelli M, et al. Cinacalcet in patients with chronic kidney disease: a cumulative meta-analysis of randomized controlled trials. PLoS Med / Public Library Science. 2013;10(4): e1001436.

17. Strippoli GF, Tong A, Palmer SC, Elder G, Craig JC. Calcimimetics for secondary hyperparathyroidism in chronic kidney disease patients. Cochrane Database Syst Rev. 2006;4:CD006254.

18. Fishbane S, Shapiro WB, Corry DB, Vicks SL, Roppolo M, Rappaport K, et al. Cinacalcet $\mathrm{HCl}$ and concurrent low-dose vitamin D improves treatment of secondary hyperparathyroidism in dialysis patients compared with vitamin D alone: the ACHIEVE study results. Clin J Am Soc Nephrol CJASN. 2008;3(6): 1718-25.

19. Messa P, Macário F, Yaqoob M, Bouman K, Braun J, von Albertini B, et al. The OPTIMA study: assessing a new cinacalcet (Sensipar/Mimpara) treatment algorithm for secondary hyperparathyroidism. Clin J Am Soc Nephrol CJASN. 2008;3(1):36-45.

20. Raggi P, Chertow GM, Torres PU, Csiky B, Naso A, Nossuli K, et al. The ADVANCE study: a randomized study to evaluate the effects of cinacalcet plus low-dose vitamin D on vascular calcification in patients on hemodialysis. Nephrol Dial Transplant. 2011;26(4):1327-39.

21. R.G.O. In: Hedges LV, Cooper H, editors. The Handbook of Research Synthesis, in Evaluating coding decisions. New York (NY): Russell Sage; 1994

22. Higgins JP, Thompson SG, Spiegelhalter DJ. A re-evaluation of randomeffects meta-analysis. J R Stat Soc Ser A Stat Soc. 2009;172(1):137-59.

23. Akl EA, Sun X, Busse JW, Johnston BC, Briel M, Mulla S, et al. Specific instructions for estimating unclearly reported blinding status in randomized trials were reliable and valid. J Clin Epidemiol. 2012;65(3):262-7.

24. Atkins D, Best D, Briss PA, Eccles M, Falck-Ytter Y, Flottorp S, et al. Grading quality of evidence and strength of recommendations. BMJ. 2004;328(7454):1490.

25. Guyatt GH, Oxman AD, Vist GE, Kunz R, Falck-Ytter Y, Alonso-Coello P, et al. GRADE: an emerging consensus on rating quality of evidence and strength of recommendations. BMJ. 2008;336(7650):924-6.

26. Guyatt $G H$, Oxman AD, Schünemann HJ, Tugwell P, Knottnerus A. GRADE guidelines: a new series of articles in the Journal of Clinical Epidemiology. J Clin Epidemiol. 2011;64(4):380-2.

27. Guyatt GH, Oxman AD, Kunz R, Woodcock J, Brozek J, Helfand M, et al. GRADE guidelines: 7. Rating the quality of evidence-inconsistency. J Clin Epidemiol. 2011;64(12):1294-302.

28. Guyatt GH, Oxman AD, Kunz R, Brozek J, Alonso-Coello P, Rind D, et al. GRADE guidelines 6. Rating the quality of evidence-imprecision. J Clin Epidemiol. 2011;64(12):1283-93.

29. Guyatt GH, Oxman AD, Kunz R, Woodcock J, Brozek J, Helfand M, et al. GRADE guidelines: 8. Rating the quality of evidence-indirectness. J Clin Epidemiol. 2011;64(12):1303-10.

30. Guyatt GH, Oxman AD, Montori V, Vist G, Kunz R, Brozek J, et al. GRADE guidelines: 5. Rating the quality of evidence-publication bias. J Clin Epidemiol. 2011;64(12):1277-82

31. Akl EA, Johnston BC, Alonso-Coello P, Neumann I, Ebrahim S, Briel M, et al. Addressing dichotomous data for participants excluded from trial analysis: a guide for systematic reviewers. PLoS One. 2013;8(2):e57132

32. Guyatt GH, Oxman AD, Santesso N, Helfand M, Vist G, Kunz R, et al. GRADE guidelines: 12. Preparing summary of findings tables-binary outcomes. J Clin Epidemiol. 2013:66(2):158-72. 